\title{
The Evolution of Insect Visual Opsin Genes With Specific Consideration of the Influence of Ocelli and Life History Traits
}

Quentin Guignard ( $\square$ quentin.guignard@fabi.up.ac.za )

University of Pretoria

Jeremy D. Allison

University of Pretoria

Bernard Slippers

University of Pretoria

\section{Research Article}

Keywords: Opsin evolution, ocelli, colour vision

Posted Date: July 29th, 2021

DOI: https://doi.org/10.21203/rs.3.rs-701324/v1

License: (c) (i) This work is licensed under a Creative Commons Attribution 4.0 International License.

Read Full License

Version of Record: A version of this preprint was published at BMC Ecology and Evolution on January 7th, 2022. See the published version at https://doi.org/10.1186/s12862-022-01960-8. 


\section{Abstract}

Background: Visual opsins are expressed in the compound eyes and ocelli of insects and enable light detection. Three distinct phylogenetic groups of visual opsins are found in insects, named long (LW), short (SW) and ultraviolet (UV) wavelength sensitive opsins. Recently, the LW group was found to be duplicated into the ancestral LW2b and the more recent LW2a opsins. The expression of LW2b opsins is ocelli specific in some insects (e.g., bees, cricket, scorpion flies), but the gene was absent in other orders possessing three or less ocelli (e.g., dragonflies, beetles, moth, bugs). In flies, two LW2b homologs have been characterised, with one expressed in the ocelli and the other in the compound eyes. To date, it remains unclear which evolutionary forces have driven gains and losses of LW opsins in insects. Here we take advantage of the recent rapid increase in available sequence data from insect genomes to characterize the phylogenetic relationships of 1000 opsin sequences in 18 orders of Insects. The resulting phylogeny discriminates between four main groups of opsins, and onto this phylogeny we mapped relevant morphological and life history traits.

Results: Our results demonstrate a conserved LW2b opsin only present in insects with three ocelli. Only two taxa (flies and dragonflies) possess more than one LW2b opsin, likely linked to their life history. In flies, we hypothesize that the duplication of the LW2b opsin occurred after the transition from aquatic to terrestrial larvae. During this transition, higher flies (Brachycera) lost a copy of the LW2a opsin, still expressed in the compound eyes of lower flies (Nematocera). In higher flies, the LW2b opsin has been duplicated and expressed in the compound eyes while the ocelli and the LW2b opsin were lost in lower flies. In dragonflies, specialisation of the visual system likely drove the diversification of visual opsins.

Conclusion: The presence of the LW2b opsin in insects possessing three ocelli suggests a role in flight. This study provides the most complete view of the evolution of visual opsin genes in insects yet, and provides new insight into the influence of ocelli and life history traits on opsin evolution in insects.

\section{Background}

The visual system is vital for the survival of insects (1). Visual cues and signals mediate the location and acquisition of resources, mates and avoidance of danger (2). Insects perceive light primarily with three main organs; compound eyes and ocelli in the adult stage and stemmata in the larval stage. Insects recognize different colours $(3,4)$ and the discrimination between different colours is linked to the diversity of opsins present in the photoreceptive cells $(1,5)$. Opsins are conserved proteins and group into three major clades linked to the wavelength they are most sensitive to $(6,7)$. The three groups are known as long $(\sim 530 \mathrm{~nm})$, short $(\sim 440 \mathrm{~nm})$ and ultraviolet $(\sim 350 \mathrm{~nm})$ wavelength (LW, SW and UV, respectively) opsins $(2,8)$.

Insects usually possess at least one copy of the LW, SW and UV opsins. Lacking a copy of one or more LW, SW or UV opsins has often been linked to a particular life history or biology. For example, the Neuropteroidea (Strepsiptera, Coleoptera, Raphidioptera, Neuroptera) and the American cockroach 
Periplaneta americana have lost their SW opsin and the corresponding blue photoreceptor, likely due to their ancestor being nocturnal $(7,9)$. The number of visual opsins was found to be reduced in three subterranean beetles; Neobidessodes gutteridgei and Paroster macrosturtensis have lost their visual opsins completely, while Limbodessus palmulaoides has lost all but the LW opsin (10). Conversely, duplication of visual opsins in dragonflies (Odonata) has been linked to the prominent size of their eyes and the role of vision in the ecology (i.e., prey location and capture) of this order (11).

Two distinct groups of LW opsins differentially expressed between the ocelli and the compound eyes have previously been identified. A homolog of the LW opsin was found to be ocelli specific in bees (12), scorpionflies (13), the cricket Gryllus bimaculus (14), the cockroach P. americana (15), dragonflies (11) and flies $(16,17)$. Ocelli specific opsins evolved at a slower rate than the other LW opsins in bees and formed a separate clade from the rest of LW opsin sequences from the same order $(12-14,18)$. Recent phylogenetic analyses focused on the Pancrustacea including a few insect species and could separate these two clades of LW opsins in insects, namely LW2a and LW2b (19). The LW2b group contained most of the previously described ocelli specific opsins and LW2a most of the LW opsins expressed in the compound eyes. However, both groups contained ocelli specific opsins and the link between the two groups of LW opsins and the presence or absence of ocelli in insects remained unclear.

The link between the LW2b opsin and the ocelli remains poorly understood. In flies, two LW2b homologs have been characterised, with one being expressed in the ocelli and the other one in the compound eyes and the larval stemmata $(16,17)$. In dragonflies, phylogenetic analyses could not distinguish between the ocelli specific opsin and the rest of the opsins expressed in the compound eyes and in the larvae (11). In Hymenoptera, one exception was found where the LW2b opsin was expressed in the compound eyes instead of the ocelli in a fig pollinator (18). No LW2b opsins were described in some insects possessing ocelli, such as mayflies (20), beetles (9) and true bugs, moths and butterflies (21). To date, it is uncertain if the LW2b is missing in some groups of insects due to a lack of data or a gene loss.

The increased availability of insect genomes (GenBank (22), I5K (23), VectorBase (24), Flybase (25), Hymenopteran Genome Database (26)) offers a large amount of data that remains unexplored with respect to questions related the evolution of insect opsin genes. In this study, we characterize the occurrence and the phylogenetic relationship of all available sequences of visual opsins from public data. Specifically, we i) identified, extracted and compiled data sets of DNA sequences of the visual opsin genes from gene sequences or genomes from 18 insect orders, ii) determined the phylogenetic relationships of the extracted visual opsin gene sequences, iii) link this more complete view of opsin phylogenetic groups to classifications of visual opsin genes, and iv) consider how the loss of ocelli and key life history traits may have shaped the evolution of opsin genes.

\section{Results}


This study found a total of 1000 insect visual opsin sequences of 340 amino acids in length after alignment (Table S1). These opsins came from 18 orders of insects, including 36 sub-orders, 89 families and 218 species. Most of the data collected was from RNA or DNA extractions. In some cases, the available set of opsins was incomplete as the objective of the study that generated the data was to target specific visual opsins. The maximum-likelihood phylogenetic tree (Figure 1) of the visual opsins contained three well separated ( $\mathrm{SH}$-alrt $\geq 0.8$ / UFBoot $\geq 0.95$ ) major clades that corresponded to the LW, SW and UV visual opsin genes. The LW, SW and UV opsin clades contained 565, 187 and 248 opsin sequences, respectively.

A copy of the LW2b opsin (Figure 1, red nodes) was found in nine insect orders including the Diptera (Brachycera), Hymenoptera, Raphidioptera, Trichoptera, Mecoptera, Ephemeroptera, Odonata, Orthoptera and Blattodea. The LW2b opsin lineage contains the ocelli specific opsins found in five species of Hymenoptera, all Odonata ( $L W D$ and $L W E$ ), the field cricket G. bimaculus, four Mecoptera, the cockroach $P$. americana and the common fruit fly Drosophila melanogaster(Rh2). A duplication of the LW2b opsin was found in all the Diptera (named Rh1 and only found in Brachycera), except for $D$. suzukii and Glossina morsitans and in all Odonata (LWB-C-F).

A total of 27 clades of LW2a opsin gene sequences were found (Figure 1, green nodes). All LW2a opsin genes group together within the orders Lepidoptera, Trichoptera, Hymenoptera, Mecoptera, Siphonaptera, Neuroptera, Raphidioptera, Phasmatodea, Odonata (LWA), Ephemeroptera and Psocodea. Two clades or more were found in Coleoptera, Hemiptera, Orthoptera, Blattodea, Thysanoptera, Diptera and Strepsipera. The LW2a opsins of Hemiptera divided into two poorly supported clades; one containing the Heteroptera suborder and the second one containing the Fulgomorpha, Cicadomorpha and Sternorrhyncha suborders. The LW2a opsins of Diptera are divided into two clades, both well supported (SH-alrt $\geq 0.8$ / UFBoot $\geq$ 0.95). The first Dipteran LW2a opsin clade contained the Rh6/GPROP7 opsin present in all the Dipteran suborders. The second LW2a opsin clade of Diptera contained the GPROP1-6-like opsins only present in the suborder of Culicomorpha and Psychodomorpha. The Buprestidae family formed a well-supported clade ( $\mathrm{SH}$-alrt $\geq 0.8$ / UFBoot $\geq 0.95$ ) separate from the rest of the Coleoptera. The SW opsin group is divided into nine clades containing species from the same order or higher ranks. The UV opsin is divided into 20 clades where species from the same order group together, with the exception of Lepidoptera, Strepsiptera and Neuroptera that grouped with other orders.

All species that had a copy of the LW2b opsin also possessed three ocelli in at least one morph, life stage or caste (Figure 2). Three ocelli and one copy of the LW2b opsin was found in the Ephemeroptera (except Epeorus sp. EP006), Odonata, Orthoptera (except Locusta migratoria, Dianemobius nigrofasciatus, and Schistocerca gregaria), Hymenoptera (except Chrysis viridula, Camponotus atriceps, Cataglyphis bomb

All species that had a copy of the LW2b opsin also possessed three ocelli in at least one morph, life stage or caste (Figure 2). Three ocelli and one copy of the LW2b opsin was found in the Ephemeroptera (except Epeorus sp. EP006), Odonata, Orthoptera (except Locusta migratoria, Dianemobius nigrofasciatus, and Schistocerca gregaria), Hymenoptera (except Chrysis viridula, Camponotus atriceps, Cataglyphis 
bombycinus, Cerapachys biroi, and Tenthredo koehleri) and the one species of Trichoptera (Limnephilus lunatus). In the Raphidioptera, Blattodea and Diptera a copy of the LW2b opsin was found within families possessing three ocelli. No copy of the LW2b was extracted from Mecoptera (Nannochoristidae) larvae, the one Thysanoptera (Frankliniella occidentalis) examined and in ant workers. The presence of three ocelli varied within Hemiptera and Neuroptera, but the LW2b opsin was never found.

cinus, Cerapachys biroi, and Tenthredo koehleri) and the one species of Trichoptera (Limnephilus lunatus). In the Raphidioptera, Blattodea and Diptera a copy of the LW2b opsin was found within families possessing three ocelli. No copy of the LW2b was extracted from Mecoptera (Nannochoristidae) larvae, the one Thysanoptera (Frankliniella occidentalis) examined and in ant workers. The presence of three ocelli varied within Hemiptera and Neuroptera, but the LW2b opsin was never found.

All species possessed one copy of the LW2a opsin, with the exception of Camponotus rufipes and Microphotus sp. Multiple copies of the LW2a opsin were found in the order Thysanoptera, in the sub-order Culicomorpha (Diptera), in the families Psyllidae, Gerridae, Lygaeidae and Pentatomidae (Hemiptera), Mengenillidae and Xenidae (Strepsiptera), Buprestidae (Coleoptera), Noctuidae, Nymphalidae and Papilionidae (Lepidoptera).

The SW opsin was identified in 55 families of insects, but not found in the Phasmatodea, Blattodea, Psocodea, Raphidioptera, Neuroptera, Strepsiptera, Coleoptera and Siphanoptera, in the sub-orders of Fulgomorpha and Heteroptera (Hemiptera), Psychodomorpha (Diptera) and in the families Siricidae (Hymenoptera) and Tephritidae (Diptera). Duplication of the SW opsin was found in all the Palaeoptera (Odonata and Ephemeroptera) families except for one species of Calopterygidae (Odonata). Within the 32 families where the LW2b opsin was found, 27 also possess a copy of a SW opsin.

The UV opsin was found in every order except for Trichoptera and Siphanoptera. Duplication of the UV opsin occurred in the families Baetidae (Ephemeroptera), Aphididae and Delphacidae (Hemiptera), Raphidiidae (Raphidioptera), Carabidae and Buprestidae (Coleoptera), Nymphalidae and Crambidae (Lepidoptera) and Drosophilidae and Tephritidae (Diptera).

\section{Discussion}

This study used 1000 visual opsins from 18 insect orders to determine the phylogenetic relationships among insect opsins. It provides the most comprehensive analysis of the evolution of visual opsin genes in insects to date. The LW2b opsin was described in new insect orders and is shown to only occur in insects with three ocelli. Some insects with three ocelli and all insects with less than three ocelli were found to lack the LW2b opsin. Our phylogenetic tree is the first to strongly link all but one of the previously described ocelli specific opsins in the LW2b opsin group. Dragonflies and flies were the only insects to possess more than one copy of the LW2b opsin, likely linked to their life history traits.

The presence of a LW2b opsin in insects with thee ocelli adds evidence to its hypothesised function in insect flight. All but one ocelli specific opsin previously described (11-17) belong to the LW2b opsin 
group in our trees, confirming that LW2b opsins are likely ocelli specific in most insects. The LW2a and LW2b appeared after the radiation of hexapods and are not present in the Crustacea $(19,27)$. Two important morphological differences that distinguish Insecta from Crustacea are the fused median ocellus (28) and the ability to fly in most adults (29). Three ocelli are primarily found in insects that are strong fliers (30) and facilitate detection of horizon tilt and a fast head and body reaction to altitude changes (31). New flight capabilities likely drove the ocelli specific LW2b opsin to adapt to its function in flying insects.

The LW2b opsin gene was commonly found in insects together with a SW opsin gene. The loss of SW opsin was found to be linked with a nocturnal lifestyle in the American cockroach $P$. americana (15) and the praying mantis Tenoda sinensi (32), adults not feeding such as Sirex noctilio (33, in press) or due to a nocturnal ancestor in the Neuropteroidae (9). Most LW2b opsins found in insect ocelli absorb in the green part of the light spectra, and are often expressed with an UV opsin $(14,32,34,35)$. Diurnal insects use the contrast between the green foliage and UV sky landscape as a primary source of information for landmark navigation (36). The co-expression of the SW and LW2b opsins suggests an important role of the LW2b opsin for daytime activities, perhaps a role in flight stabilisation.

The LW2b opsin likely has alternative functions other than flight stabilisation as it was found in some flightless insects. A LW2b opsin gene was found in the nocturnal and non-flying American cockroach. In addition to flight stabilisation, ocelli were found to be linked with celestial navigation (37-39), light polarisation (39), circadian rhythm $(18,40)$ or to detect variation of light intensity that mediates flight activity (41). Similarly, males of a fig pollinator species do not fly, spend all their life inside the fruit (in low light conditions) and do not have ocelli, yet a LW2b opsin is expressed in this species (18). In agreement with previous studies (19), opsin expression is flexible and can switch between compound eyes and ocelli, likely to adapt to functions other than flight stabilisation. Two good examples of flexibility in opsin expression are flies and dragonflies where a duplication of the LW2b opsin was found, with one homolog being ocelli-specific and one or more LW2b homologs expressed in the larvae and/or the compound eyes.

In Diptera, two LW2b homologs were only found in higher flies (Brachycera) and no LW2b opsins were found in lower flies (Culicomorpha and Psychodomorpha). The ancestor of Diptera was believed to have three ocelli and aquatic larvae similar to the lower dipteran sub-orders (42). The absence of LW2b opsin in lower flies is most likely due to the lack of ocelli in these sub-orders. The various LW2a homologs found in the lower flies Aedes aegypti (GPROP1-6-like gene) are first expressed in the aquatic larvae and then in the compound eyes of the adults $(43,44)$. These LW2a homologs are absent in higher flies, but the ocelli specific LW2b (Rh2) was conserved with the three ocelli. It is likely that higher flies duplicated the conserved LW2b Rh2 opsin expressed in their ocelli to create the LW2b homolog $R h 1$, expressed both in the terrestrial larvae and in the adult compound eye (17). In agreement with previous studies (14), the split between $R h 1$ and $R h 2$ in our phylogenetic tree is more recent than the split between the different Dipteran sub-orders, supporting the interpretation that the $R h 1$ appeared after the emergence of flies. Therefore, the historically named blue-green opsin group of Diptera can be considered as a LW2b opsin 
which has undergone a duplication, likely driven by the transition from an aquatic to a terrestrial larval life style.

In dragonflies, most of the LW opsin expansion resulted in the diversification of numerous LW2b opsins. Our results correlate with previous studies showing a segregation between two LW opsin groups in dragonflies (19). The LW2a and LW2b genes in the dragonfly Ladona fulva are found in two different genomic regions (11). The authors of that study demonstrated that only one to two LW2b opsins were found to be ocelli specific while the other LW2b homologs were expressed in the compound eyes and larval eyes. The high demand for visual performance and flight capabilities in dragonflies, in addition to the aquatic larvae, likely drove the diversification of opsins in this group. In various insects, it is the LW2a opsin that is duplicated and adapted to the specific needs of each species $(9,20,21,45)$. It is unclear why the LW2b opsins in dragonflies evolved and diversified instead of the LW2a.

\section{Conclusions}

This study confirmed that the LW2b opsin is well conserved among insects possessing three ocelli. The occurrence of the LW2b ocelli-specific opsin is consistent with a potential role in flight stability and/or horizon detection in flying insects. We hypothesize that life history and transition toward a terrestrial environment in fly larvae was a driving force in opsin evolution in Diptera and could explain the historically named blue-green clade unique to flies, in a broader evolutionary scenario. Similarly, dragonflies also evolved numerous homologs of the LW2b opsins likely as a part of the evolution of more accurate flight capabilities in addition to their life history traits. Many species across various insect orders possess three ocelli but no LW2b opsin was found (e.g Orthoptera, Hemiptera, Thysanoptera or Neuroptera). It is possible that this is due to a lack of data in these species, and this needs to be confirmed. If not, these species offer opportunities to further investigate the different function of the LW2b and LW2a opsins in the ocelli.

\section{Methods}

To determine the relationships among visual opsins in insects, we identified opsin DNA, RNA and protein sequences via searches of the literature $(6,7,9,11,12,14)$ and online databases ((GenBank (22), I5K (23), VectorBase (24), Flybase (25), Hymenopteran Genome Database (26)) (Table S1). We performed BLAST searches for visual opsin DNA, RNA or protein sequences using annotated genes from Apis mellifera and Drosophila melanogaster to find insect visual opsins when genomes were available. Sequences with an e-value $<10^{-40}$ were retained. We used the genomic sequences to locate opsin genes in the unpublished Sirex noctilio genome (Alisa Postma, pers. comm). The Rh7opsin was excluded from the dataset due to the uncertain role of this opsin in vision (6). The gene predictor Augustus (http://bioinf.unigreifswald.de/augustus/submission.php) was used to translate DNA sequences into amino acid sequences. Sequences of RNA were translated into amino acid sequences via MEGA7 (46). The Bos taurus rhodopsin was chosen as an outgroup (OG) for two reasons: i) its LW opsin is well studied and used as a reference to annotate amino acid sequences of opsins $(7,47)$ and ii) the bovine LW opsin 
diverged before the split between the insects LW and UV in the Celicerata-Pancrustacea ancestor allowing the bovine LW opsin to root the tree.

Amino acid sequences were aligned using MAFFT using the default parameters. After alignment, data were visually curated to remove remaining intron sequences. Aligned amino acid sequences were cut and sequences between the $\mathrm{K}^{25}$ and the $\mathrm{A}^{329}$ of the $A$. mellifera Rh1 LW (sequence annotated relative to the reference opsin) were retained. Curated and aligned sequences are available (see Availability of data and material section). The phylogenetic reconstruction was performed using IQtree v.1.4.4 (48). The most probable amino acid substitution model was found to be the $L G+F+\mid+G 4$. This model was used to build an independent maximum-likelihood tree with an ultra-fast bootstrap value of 1000 ultrafast bootstrap iterations (UFboot, Minh et al., 2013) and a 1000 Shimodaira-Hasegawa approximate likelihood-ratio test $(\mathrm{SH}-a l r t,(50))$ to assess the nodal support. Graphic representation of phylogenetic relationships was done using Figtree (V.1.4.3 http://tree.bio.ed.ac.uk/software/figtree).

The number of opsins found in each opsin group, as defined in the maximum-likelihood phylogenetic tree, were determined for each species and averaged for each family of insects. The presence or absence of three ocelli in the adult life stage of each species was determined from the literature. In instances where there was no information available for a given species, we assumed that species within families shared the same number of ocelli as adults.

\section{Abbreviation}

LW: long-wavelength sensitive opsin, SW: short-wavelength sensitive opsin, UV: ultraviolet-wavelength sensitive opsin.

\section{Declaration}

\section{Availability of data and material}

The aligned sequences and the phylogenetic trees analysed during the current study are available in the open Harvard dataverse repository at: https://dataverse.harvard.edu/dataset.xhtml? persistentld=doi:10.7910/DVN/FVLIBE.

\section{Competing of interests}

The authors declare that they have no competing interests.

\section{Funding}

This research was funded by the United States Department of Agriculture-Forest Service Forest Health Protection (USDA-FS FHP), Natural Resources of Canada (NRCan), the Tree Protection Cooperative Program (TPCP) and the DSI NRF Centre of Excellence in Plant Health Biotechnology (CPHB) in South 
Africa. The funders had no role in study design, data collection and analysis, decision to publish, or preparation of the manuscript.

\section{Author's contribution}

QG conducted the data collection and analyses; QG, JDA and BS conceived the study and wrote the manuscript. All authors have read and approved the manuscript.

\section{Acknowledgments}

We thank Alisa Postma for helping with the $S$. noctilio sequences and Dr. Xiong Peng for providing the opsin sequences of Rhopalosiphum padi. We thank Amanda Adlam for her review of an early draft of the manuscript.

\section{References}

1. Cronin TW, Johnsen S, Marshall NJ, Warrant E. Visual ecology [Internet]. 2017 [cited 2018 Jun 1]. Available from: http://dx.doi.org/10.23943/princeton/9780691151847.001.0001

2. van der Kooi CJ, Stavenga DG, Arikawa K, Belušič G, Kelber A. Evolution of insect color vision: from spectral sensitivity to visual ecology. Annu Rev Entomol. 2021;66(1):435-61.

3. Lebhardt F, Desplan C. Retinal perception and ecological significance of color vision in insects. Curr Opin Insect Sci. 2017 Dec;24:75-83.

4. Wernet MF, Perry MW, Desplan C. The evolutionary diversity of insect retinal mosaics: common design principles and emerging molecular logic. Trends Genet. 2015 Jun;31(6):316-28.

5. Kelber A, Vorobyev M, Osorio D. Animal colour vision - behavioural tests and physiological concepts. Biol Rev Camb Philos Soc. 2003 Feb;78(1):81-118.

6. Feuda R, Marlétaz F, Bentley MA, Holland PWH. Conservation, duplication, and divergence of five opsin genes in insect evolution. Genome Biol Evol. 2016 Feb;8(3):579-87.

7. Lord NP, Plimpton RL, Sharkey CR, Suvorov A, Lelito JP, Willardson BM, et al. A cure for the blues: opsin duplication and subfunctionalization for short-wavelength sensitivity in jewel beetles (Coleoptera:

Buprestidae). BMC Evol Biol [Internet]. 2016 Dec [cited 2016 Sep 23];16(1). Available from:

http://bmcevolbiol.biomedcentral.com/articles/10.1186/s12862-016-0674-4

8. Briscoe AD, Chittka L. The evolution of color vision in Insects. Annu Rev Entomol. 2001;46(1):471-510.

9. Sharkey CR, Fujimoto MS, Lord NP, Shin S, McKenna DD, Suvorov A, et al. Overcoming the loss of blue sensitivity through opsin duplication in the largest animal group, beetles. Sci Rep [Internet]. 2017 Dec [cited 2018 Mar 29];7(1). Available from: http://www.nature.com/articles/s41598-017-00061-7 
10. Tierney SM, Cooper SJB, Saint KM, Bertozzi T, Hyde J, Humphreys WF, et al. Opsin transcripts of predatory diving beetles: a comparison of surface and subterranean photic niches. R Soc Open Sci. 2015 Jan 1;2(1):140386.

11. Futahashi R, Kawahara-Miki R, Kinoshita M, Yoshitake K, Yajima S, Arikawa K, et al. Extraordinary diversity of visual opsin genes in dragonflies. Proc Natl Acad Sci. 2015 Mar 17;112(11):E1247-56.

12. Spaethe J, Briscoe AD. Early duplication and functional diversification of the opsin gene family in Insects. Mol Biol Evol. 2004 Aug 1;21(8):1583-94.

13. Böhm A, Meusemann K, Misof B, Pass G. Hypothesis on monochromatic vision in scorpionflies questioned by new transcriptomic data. Sci Rep [Internet]. 2018 Dec [cited 2019 Jan 7];8(1). Available from: http://www.nature.com/articles/s41598-018-28098-2

14. Henze MJ, Dannenhauer K, Kohler M, Labhart T, Gesemann M. Opsin evolution and expression in arthropod compound eyes and ocelli: insights from the cricket Gryllus bimaculatus. BMC Evol Biol. 2012 Aug 30;12:163.

15. French AS, Meisner S, Liu H, Weckstrom M, Torkkeli PH. Transcriptome analysis and RNA interference of cockroach phototransduction indicate three opsins and suggest a major role for TRPL channels. Front Physiol [Internet]. 2015 [cited 2020 May 10];6. Available from: https://www.frontiersin.org/articles/10.3389/fphys.2015.00207/full

16. Friedrich $M$, Wood EJ, Wu M. Developmental evolution of the insect retina: insights from standardized numbering of homologous photoreceptors. J Exp Zoolog B Mol Dev Evol. 2011 Nov 15;316B(7):484-99.

17. Pollock JA, Benzer S. Transcript localization of four opsin genes in the three visual organs of Drosophila; RH2 is ocellus specific. Nature. 1988 Jun;333(6175):779-82.

18. Wang B, Xiao J-H, Bian S-N, Niu L-M, Murphy RW, Huang D-W. Evolution and expression plasticity of opsin genes in a Fig pollinator, Ceratosolen solmsi. Crandall KA, editor. PLoS ONE. 2013 Jan 16;8(1):e53907.

19. Henze MJ, Oakley TH. The dynamic evolutionary history of Pancrustacean eyes and opsins. Integr Comp Biol. 2015 Nov;55(5):830-42.

20. Almudi I, Vizueta J, Wyatt CDR, de Mendoza A, Marlétaz F, Firbas PN, et al. Genomic adaptations to aquatic and aerial life in mayflies and the origin of insect wings. Nat Commun. 2020 May 26;11(1):2631.

21. Briscoe AD. Functional diversification of lepidopteran opsins following gene duplication. Mol Biol Evol. 2001 Dec 1;18(12):2270-9.

22. Benson DA, Cavanaugh M, Clark K, Karsch-Mizrachi I, Lipman DJ, Ostell J, et al. GenBank. Nucleic Acids Res. 2013 Jan 1;41(D1):D36-42. 
23. Poelchau M, Childers C, Moore G, Tsavatapalli V, Evans J, Lee C-Y, et al. The i5k Workspace@NALenabling genomic data access, visualization and curation of arthropod genomes. Nucleic Acids Res. 2015 Jan 28;43(D1):D714-9.

24. Giraldo-Calderón GI, Emrich SJ, MacCallum RM, Maslen G, Dialynas E, Topalis P, et al. VectorBase: an updated bioinformatics resource for invertebrate vectors and other organisms related with human diseases. Nucleic Acids Res. 2015 Jan 28;43(D1):D707-13.

25. dos Santos G, Schroeder AJ, Goodman JL, Strelets VB, Crosby MA, Thurmond J, et al. FlyBase: introduction of the Drosophila melanogaster Release 6 reference genome assembly and large-scale migration of genome annotations. Nucleic Acids Res. 2015 Jan 28;43(D1):D690-7.

26. Elsik CG, Tayal A, Diesh CM, Unni DR, Emery ML, Nguyen HN, et al. Hymenoptera genome database: integrating genome annotations in HymenopteraMine. Nucleic Acids Res. 2016 Jan 4;44(D1):D793-800.

27. Kashiyama K, Seki T, Numata H, Goto SG. Molecular characterization of visual pigments in branchiopoda and the evolution of opsins in Arthropoda. Mol Biol Evol. 2009 Feb 1;26(2):299-311.

28. Sabat D, Priyadarsini S, Mishra M. Understanding the structural and developmental Aspect of simple eye of Drosophila: the ocelli. J Cell Signal. 2016 Mar 21;1(2):1-10.

29. Misof B, Liu S, Meusemann K, Peters RS, Donath A, Mayer C, et al. Phylogenomics resolves the timing and pattern of insect evolution. Science. 2014 Nov 7;346(6210):763-7.

30. Goodman LJ. The structure and function of the insect dorsal ocellus. In: Beament JWL, Treherne JE, Wigglesworth VB, editors. Advances in Insect Physiology [Internet]. Academic Press; 1970 [cited 2020 May 18]. p. 97-195. Available from:

http://www.sciencedirect.com/science/article/pii/S0065280608602416

31. Taylor GK, Krapp HG. Sensory systems and flight stability: what do insects measure and why? In: Casas J, Simpson SJ, editors. Advances in Insect Physiology [Internet]. Academic Press; 2007 [cited 2020 May 10]. p. 231-316. (Insect Mechanics and Control; vol. 34). Available from: http://www.sciencedirect.com/science/article/pii/S0065280607340058

32. Sontag C. Spectral sensitivity studies on the visual system of the praying mantis, Tenodera sinensis. J Gen Physiol. 1971 Jan 1;57(1):93-112.

33. Quentin Guignard, Johannes Spaethe, Bernard Slippers, Martin Strube-Bloss, Jeremy D. Allison. Evidence for UV-green dichromacy in the basal hymenopteran Sirex noctilio (Siricidae). Sci Rep [Internet]. 2021 Feb 23; Available from: https://doi.org/10.21203/rs.3.rs-271270/v1

34. Chappell RL, DeVoe RD. Action spectra and chromatic mechanisms of cells in the median ocelli of dragonflies. J Gen Physiol. 1975 Apr 1;65(4):399-419. 
35. Velarde RA, Sauer CD, O. Walden KK, Fahrbach SE, Robertson HM. Pteropsin: A vertebrate-like nonvisual opsin expressed in the honey bee brain. Insect Biochem Mol Biol. 2005 Dec;35(12):1367-77.

36. Möller R. Insects could exploit UV-Green contrast for landmark navigation. J Theor Biol. 2002 Feb;214(4):619-31.

37. Schwarz S, Albert L, Wystrach A, Cheng K. Ocelli contribute to the encoding of celestial compass information in the Australian desert ant Melophorus bagoti. J Exp Biol. 2011 Mar 15;214(6):901-6.

38. Schwarz S, Wystrach A, Cheng K. A new navigational mechanism mediated by ant ocelli. Biol Lett. 2011 Dec 23;7(6):856-8.

39. Taylor GJ, Ribi W, Bech M, Bodey AJ, Rau C, Steuwer A, et al. The dual function of orchid bee ocelli as revealed by X-ray Mmcrotomography. Curr Biol. 2016 May 23;26(10):1319-24.

40. Rence BG, Lisy MT, Garves BR, Quinlan BJ. The role of ocelli in circadian singing rhythms of crickets. Physiol Entomol. 1988;13(2):201-12.

41. Eaton JL, Tignor KR, Holtzman GI. Role of moth ocelli in timing flight initiation at dusk. Physiol Entomol. 1983;8(4):371-5.

42. Wiegmann BM, Trautwein MD, Winkler IS, Barr NB, Kim J-W, Lambkin C, et al. Episodic radiations in the fly tree of life. Proc Natl Acad Sci U S A. 2011;108(14):5690-5.

43. Hu X, Leming MT, Metoxen AJ, Whaley MA, O’Tousa JE. Light-mediated control of rhodopsin movement in mosquito photoreceptors. J Neurosci. 2012 Oct 3;32(40):13661-7.

44. Rocha M, Kimler KJ, Leming MT, Hu X, Whaley MA, O'Tousa JE. Expression and light-triggered movement of rhodopsins in the larval visual system of mosquitoes. J Exp Biol. 2015 May 1;218(9):138692.

45. Briscoe AD. Six opsins from the butterfly Papilio glaucus: molecular phylogenetic evidence for paralogous origins of red-sensitive visual pigments in insects. J Mol Evol. 2000 Aug;51(2):110-21.

46. Kumar S, Stecher G, Tamura K. MEGA7: molecular evolutionary genetics analysis version 7.0 for bigger datasets. Mol Biol Evol. 2016 Jul 1;33(7):1870-4.

47. Porter ML, Blasic JR, Bok MJ, Cameron EG, Pringle T, Cronin TW, et al. Shedding new light on opsin evolution. Proc R Soc B. 2012 Jan 7;279(1726):3-14.

48. Nguyen L-T, Schmidt HA, von Haeseler A, Minh BQ. IQ-TREE: a fast and effective stochastic algorithm for estimating maximum-likelihood phylogenies. Mol Biol Evol. 2015 Jan;32(1):268-74.

49. Minh BQ, Nguyen MAT, von Haeseler A. Ultrafast approximation for phylogenetic bootstrap. Mol Biol Evol. 2013 May 1;30(5):1188-95.

Page 12/15 


\section{Figures}

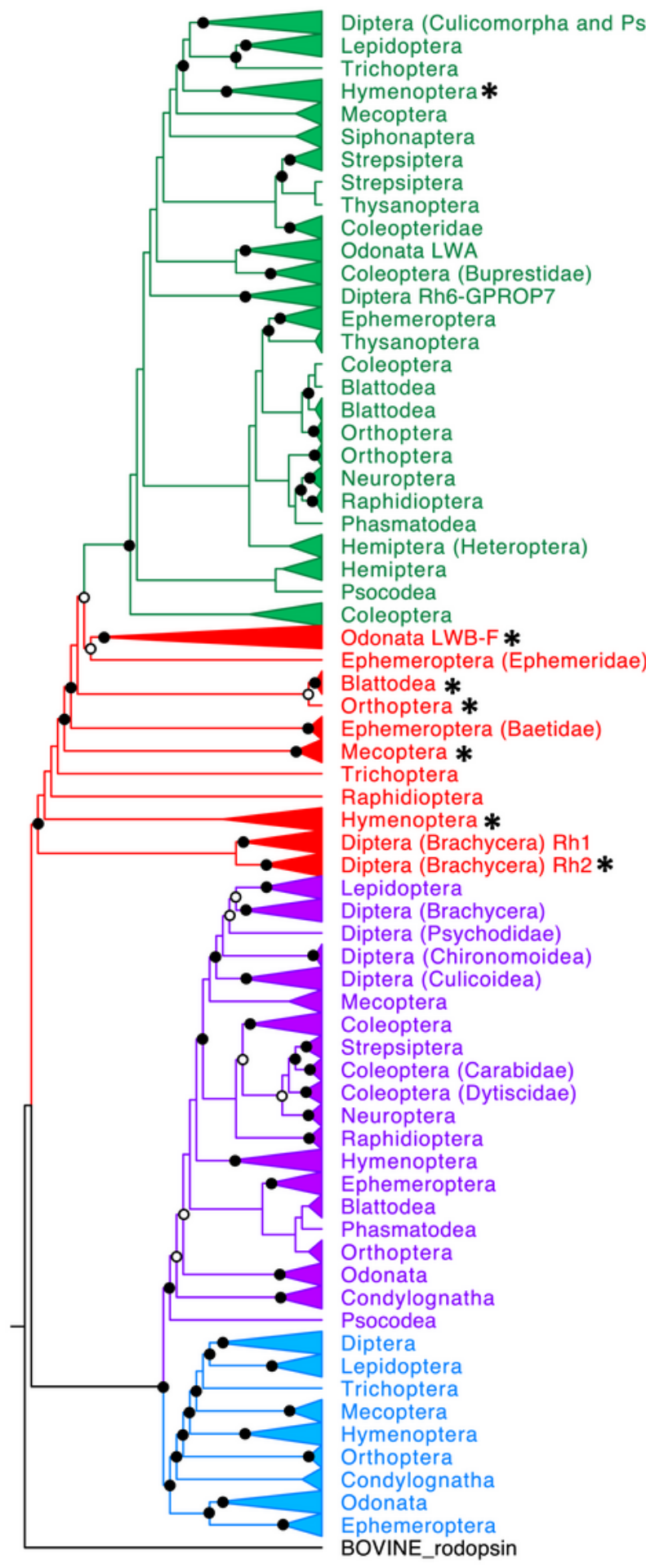

\begin{tabular}{|l|}
\hline Opsin group \\
- LW2a \\
LW2b \\
UV \\
SW \\
Bootstrap \\
- SH-alrt $\geq 80 \%$ and \\
UF-bootstrap $\geq 95 \%$ \\
O SH-alrt $\geq 80 \%$ and \\
UF-bootstrap $\geq 90 \%$ \\
* Clade containing \\
ocelli specific LW \\
opsin
\end{tabular}


Maximum-likelihood tree of 1000 insect visual opsins sequences. The LW2b, LW2a, SW and UV opsins are red, green, blue and purple, respectively. Branches were collapsed to the highest rank when species grouped together within the same family, suborder or order. Node circles indicates UFbootstrap and SHalrt value, only nodes over SH-alrt support $\geq 80 \%$ and UF-bootstrap $\geq 95 \%$ (solid circles) and SH-alrt support $\geq 80 \%$ and UF-bootstrap $\geq 90 \%$ (open circle) are represented.

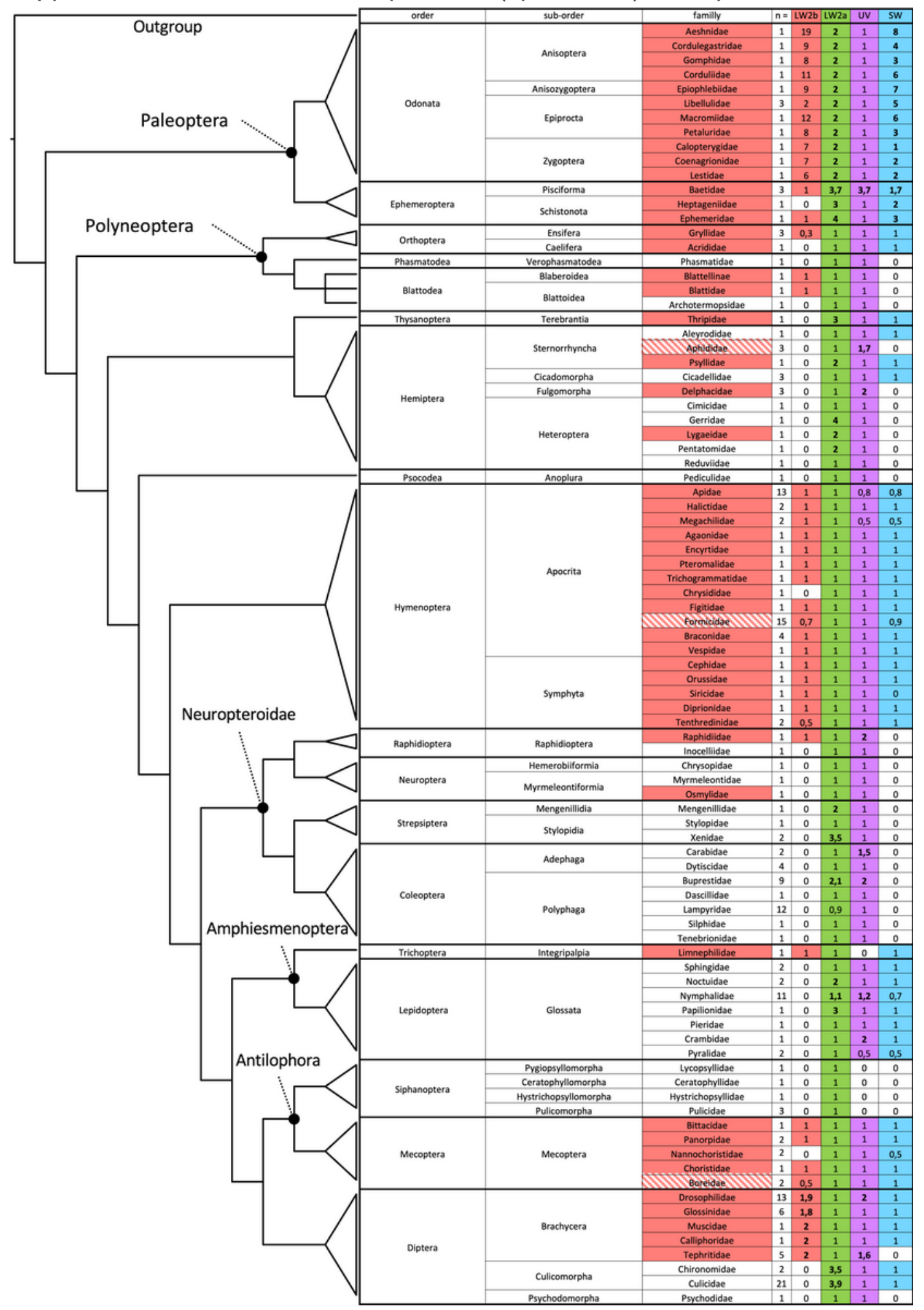

Figure 2 
Evolutionary history of the number of visual opsins in 89 families of Insects ( $n=$ number of species for each family). Number of LW2b, LW2a, SW and UV opsins were averaged per family. Boxes are highlighted in red, green, blue and purple respectively for the LW2b, LW2a, SW and UV opsins when the average number of opsin per species within each group was higher than zero. Bold numbers indicate an average number of visual opsin per species $\geq 1$. Families are highlighted or dashed in red when the presence of three ocelli in the adult stage was consistent or variable, respectively. The illustrated insect phylogeny (left) was manually coded following the insect phylogeny from Misof et al. (2014).

\section{Supplementary Files}

This is a list of supplementary files associated with this preprint. Click to download.

- SupplementarymaterialTable1.pdf 